
The Instituto Internacional de Literatura Iberoamericana was organized in 1938 in order to advance the study of Iheroamerican Literature and to promote cultural relations among the peoples of the Americas.

To this end, the Institute publishes the Revista lberoamericana tri-annually and sponsors the publication of noteworthy books by Iberoamerícan authors -in their original language and in English translation-, and of learned works and textbooks.

Members of the Institute meet in Congresses every two years and are of two types: regular members who pay $\$ 10.00$ a year, except in Iberoamérica where the fee is $\$ 3.00$, and Patron Members who pay $\$ 15.00$ or more a year.

Institutions such as universities, colleges, and libraries may become subscribers (at $\$ 7.00$ a year or $\$ 3.00$ a year in Iberoamérica), or $\$ u b$ scribing Patrons (at a minimum of $\$ 10.00$ a year) without holding membership in the Institute.

Regular nembers and subscribers receive the forthcoming issues of the Revista Iberoamericana free, but Patrons (whether Members or Subscribers) receive, in addition, the forthcoming issues of all the publications of the Institute, such as the Clásicos de América, the Proceeding of the Congresses, etc., and their names will appear in the Revista Iberoamericana at the end of the year.

\section{$\mathrm{NO}$ T I C E}

This year when you receive your Notice for renewal there will be a note explaining the different categoties of membership and the difference between members and suscribers. Please pay your dues Accordingly.

Thank you, Julio Matas Sec. Tres.

\section{SUBSCRIPTION APPLICATION}

I would like to become a subscriber to the Revista Iberoamericana. Would you please send my subsctiption to the address indicated below. I understand that I may become a member by following the steps indicated in the statues of the Instituto, which may be obtained upon request.

\begin{tabular}{|c|c|c|c|}
\hline I am enclosing: & $\begin{array}{r}10.00 \\
\$ 3.00 \\
15.00\end{array}$ & $\begin{array}{l}(\quad) \\
()\end{array}$ & $\begin{array}{l}\text { Regular subscription } \\
\text { Latin America only } \\
\text { Patron subscription } \\
\text { publications) }\end{array}$ \\
\hline ess & & & \\
\hline
\end{tabular}

Please make your checks payable to the Instituto Internacional de Literatura Iberoamericana and mail your dues to Mrs. Gloria J. Warnd 1617 C.L. University of Pittsbugh, Pittsburgh 13, Pennsylvania, U.S.A. All communications regarding the circulation and distribution of the publications of the Institute should also be addressed to Mrs. Ward. 


\section{SOCIOS Y SUSCRIPTORES}

Fl instituto Internacional de Literatura Iberoamericana se organizó en 1938 con el fin de incrementar el estudio de la Literatura Iberoamericana e intensificar las relaciones culturales entre todos los pueblos de América.

Con este fin. el Instituto publica la Revisla lberoamericana, por lo menos tres veces al año, y patrocina la publicación de obras notables de autores iberoamericanos - en el idioma original y en traducción inglesa-, y la de obras de erudición y textos de enseñanza.

Los socios del Instituto se reúnen en Congreso cada dos años, y son de dos categorías: el socio de número, cuya cuota anual es de diez dólares, excepto en Iberoamérica, donde es de sólo tres dólares, y el Socio Protector, cuya cuota es de quince dólares o más al año.

Las bibliotecas, colegios, universidades y demás instituciones que, sin ser socios, sí favorecen al Instituto, son de dos categorías: el suscriptor los países de Iberoamérica, y el Suscriptor Protector, cuya cuota es de diez dólares al año.

La Revista Iberoamericana se remite a los socios de número y a los suscriptores corrientes del Instituto, y tanto los Socios Protectores como corriente, cuya cuota anual es de siete dólares y de sólo tres dólares en los Suscriptores Protectores reciben, además de la Revista, las demás publicaciones que vayan saliendo, tales como los Clásicos de América y las Memorias, y sus nombres se publican en la Revista Iberoamericana al fin de cada año.

\section{N I TACION}

El Instituto invita cordialmente a quienes simpaticen con los fines que persigue, a que se hagan, ora socios, ora Protectores de él. Quienes así lo apoyen deben enviar su cuota anual, por adelantado, en forma de giro postal o bancario pagadero al Instituto Internacional de Literatura Iberoamericana $y$ por conducto de la señora Gloria Ward -CL-1617, University of Pittsburgh, Pittsburgh 13, Pennsylvania, U. S. A.-, que es la tinica persona encargada de la circulación y la distribución de las publicaciones del Instituto.

\section{TRES NUMEROS POR ANTO}

A partir de 1969 la Revista lberoamericana se publicará tres veces al año, en vez de dos, como se ha hecho hasta ahora.

Los manuscritos deberán ser enviados al Director antes de las siguientes fechas: $1^{\circ}$ de diciembre, para el primer número (enero-abril); $1^{\circ}$ de febrero, para el segundo número (mayo-agosto), y $1^{\circ}$ de mayo, para el tercer número (septiembre-diciembre).

Próximos números serán dedicados a César Vallejo, Octavio Paz, Pablo -Neruda, Julio Cortázar y otros novelistas actuales.

\section{LA DIRECCION}




\section{Revista Iberoamericana \\ Organo del Instituto Internacional \\ de Piteratura Iberoamericane}

Vol. XXXV

Enero-Abril de 1969

Núm. 67

PATROCINADA POR LA UNIVERSIDAD DE PITTSBURGH

Director

Alfredo A. Roggiano, C.L. I6r7, Universidad de Pittsburgh, Pittsburgh 13, Pennsylvania, U.S.A.

SECRETARTO-TESORERO

Julio Matas, C.L. I617, University of Pittsburgh, Pittsburgh r3, Pennsylvania, U.S.A.

JefE de CANJe

LILJAN S. DE LOZANO

Comisión Editorial (1967-1969)

Fernando Alegria, Stanford University, Palo Alto, Califormia. Enrique ANderSON IMBERT, Harvard University, Cambridge, Mass. FrANK DAUster, Rutgers University, New Brunswick, N. J. Francisco Monterde, Academia Mexicana de la Lengua, México. Allen W. Phillips, University of Texas, Austin, Texat 


\section{LITERATURA IBEROAMERICANA}

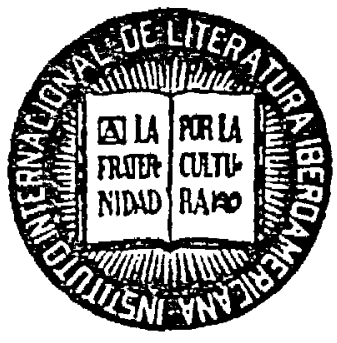

M E S A D I R E C T I A

(1967-1969)

\section{PRESIDENTE}

Kurt Levy, University of Toronto, Toronto, Canada

\section{VICEPRESIDENTES}

Jack Parker, University of Toronto, Toronto, Canadá Aníbal Sánchez-Reulet, University of California,

Los Angeles, USA.

\section{VOCALES}

John E. Englekirk, University of California, Los Angeles. Luis Leal, University of Illinois, Urbana, Illinois, USA

$$
\text { SECRETARIO-TESORERO EJECUTTVO }
$$

Julio Matas, University of Pittsburgh, Pa., USA

\section{DIRECTOR DE PUBLICACIONES .}

Alftedo A. Roggiano, Universıly of Pittsburgh, $P a_{*}, U S A$ 


\section{REVISTA IBEROAMERICANA}

\section{PROPOSITOS}

Esta REviSTA aspira a constituir, gradualmente, usa vital representación de los valores espirituales de la creciente cultura iberoamericana.

Su director y asesores quieren hacer vivo el lema que cifra el ideal adoptado por nuestro Instituto: A LA FRATERNIDAD POR LA CULTURA.

Reflejará en sus páginas una clara imagen de la literatura $v$ del pensamiento de Iberoamérica.

\section{NORMAS EDITORIALES}

La REVISTA IBEROAMERICANA sólo publicará artículos aceptados por su Director, quien será asesorado por la Comisión Editorial "Ad-hoc". Las ideas contenidas en lus artículos que se publiquen pertenecen al autor, quien será único responsable de las mismas.

Se recomienda que en los manuscritos de artículos, notas y reseñas presentados para su publicación se sigan las normas de "The MLA Style Sheet" publicado en PMLA, lxvi (1951).

La publicación de cualquier trabajo publicado en la R.I. deberá ser autorizado por el Director.

\section{CANJE Y SUSCRIPCIONES}

Todo lo referente a canje y demás intercambio de publicaciones con casas editoras, instituciones o autores deberá hacerse por intermedio del Director-Editor, $\mathrm{y}$ a tal efecto se ruega dirigirse a: Alfredo A. Roggiano, Department of Hispanic Langs., University of Pittsburgh, Pittsburgh 13, Pennsylvania, U.S.A. Todo lo referente a suscripciones, compras, órdenes de pago, etc., en que sea menester la intervención de la Tesoreria, deberá hacerse por intermedio del Secretario Ejecutivo-Tesorero, y a tal efecto se ruega escribir a: Jul1o Matas;-Department of Hispanic Langs., University of Pittsburgh, Pittsburgh 13, Pennsylvania, U.S.A. 


\title{
SOCIOS PROTECTORES
}

\author{
U.S.A.
}

Abilene Christian College R.514

Library

Attn: Miss Collie F.W. Abilene, Texas 79601

Alberto Andino 363 Twin Oaks Rd. Pittsburgh, $\mathrm{Pa}, 15243$

Alfred Owen Aldridge R-645 Comparative Literature Dept. University of Illinois

Urbana, Illinois 61801

*Allegheny College R-515

Library

Meadville, Pa.

David H. Allen R-516

Department of Spanish

University of California

Davis, California 95616

Dr. Humberto Alvarez R-043

Prof. Span. \& Span. Lit.

State Teachers College

Kirksville, Missouri 63501

Roberto M. Assardo R-65.2

Dept. of Spanish

University of California

Davis, California 95616

T. B. Avalle-Arce R-518

22 Paradise Road

Northampton, Mass. 01060

*Prof. George Ayora R-737

Dept. of Foreign Languages

Calif. State College

25800 Hillary St.

Hayward, Calif. $94 \$ 42$

C. C. Bacheller R.614

3616 Pinecrest Rd.

Vestal, New York 13850

David Ballesteros R-668

Dept. of Modern Languages

The Univ. of Oklahoma

Norman, Okla. 73069
Hal Ballew R.585

The University of Mississippi

P.O. Box 55

University, Miss. 38677

Barry College R-644

Dept. of Foreign Languages

11300 N.E. 2nd Ave.

Miami, Fla. 33161

*Prof. Rubén Benítez R-820

11097 Strathmore Drive

Los Angeles, California 90024

Rodney V. Bodden R-521

Dept. of Romance Languages

University of Washington

Seattle, Washington 98105

Florence Bonhard R-522

83 Fremont Place

Los Angeles, California 90005

Wayne S. Bowen R-523

Dept. of Foreign Langs.

Fresno State College

Fresno, California 93726

W. J. Bruner R-524

Modern Language Department

William Jewell College

Liberty, Missouri

Luz Campana de Watts R-525

5814 Summit View Ave.

Los Angeles 42, Calif.

José María Carranza R-101

331 Devonshite St.

Pittsburgh, Pa. 15213

Humbertina Carrasco R.552

3423 N. Janssen Ave.

Chicago, Ill. 60657

Prof. Boyd G. Carter R-528

1108 W. Steward Road

Columbia, Mo. $6 \$ 201$

Homero Castillo R-530

622 Sproul Hall

University of California

Davis, California 95616
Othón Castillo R.532

P.O. Box 5579

Metropolitan Station

Los Angeles 55, California

Rebecca Catz R-104

300 El Camino Drive

Beverly Hills, Calif. 90212

Eugenio Chang-Rodriguez

Box 33 Queens College

City University of New York

Flushing 67, New York

Clatemont College R-533

The Honnold Library

Periodicals Department

Claremont, California 91711

H. Logan Cobb R-534

Dept. of Foreign Lang.

Eastern Illinois University

Charleston, Illinois 61920

Colorado State College R.535

Library

Greeley, Colorado 80631

Alyce G. DeKuehne R-557

Dept. of Spanish \& Port.

University of California

Santa Barbara, California.

Dra. Angela B. Dellepiane

R-536

Dept. of Romance Languages

City College

133rd at Convent Avenue

New York, N.Y. 10031

Pauline B. Deuel R-735

Department of Spanish

Wellesley College

Wellesley, Mass. 02181

Robert P. Dixon R-658

Department of Spanish

300 Broadway (1414)

Essex Catholic H.S.

Newark, N.J. 07104 
Pedro M. Duelo R-537

1002 37th St.

San Pedro, California

*Prof. Julio Durán-Cerda R611

University of Iowa

Dept. of Romance Langs.

Iowa City, Iowa 52240

Peter G. Earle R-538

Box 42ch

Philadelphia, Pa. 19104

Fastern New Mexico Univ. R-539

Library

Portales, New Mex, 88130

York S. Febres R-806

6100 Walnut St.

Pittsburgh, Pa. 15206

Felina Ferragut R-255

Department of Spanish

Barnard College

Columbia Univ., N.Y. 10027

Prof. José Ferrer-Canales R527

Box $841^{* *}$

Howard University

Joseph A. Feustle R-152

11 Linden Terrace

Pikesville, Maryland 21208

Dr. Esperanza FigueroaAmaral

Elmira College

Elmira, N. 14901

*Dr. Nava E. Filer R-621

175 W. 93rd Street

New York, N.Y. 10025

Dr. Orlitio Fuentes R-543

Dept. of Foreign Languages

Stoke Hall

Durham, New Hampshire

Jaime Giordano R-171

Dept. of Romance Languages

State University of N.Y.

Stonybrook, New York

Prof. A. A. Greene R-666

Foreign Languages

Department

Trinity University

San Antonio, Texas 78212
*Alberto Gutiérrez de la Solana

640 W. 231st. St. R-508

Apt. 3B

New York, N.Y. 10463

Juana Amelia Hernández R-547

Hood College

Frederick, Maryland 21702

*Carole Adele Holdworth R-608

831 South Harvey Ave.

Oak Park, Ill. 60304

Howard University R-548

Library-Serials Dept.

Washington, D.C. 20001

University of Illinois R-549

Program in Comparative Lit.

Urbana, Illinois 61801

Indiana University of $\mathrm{Pa}$. R-550

Library

Indiana, Penna. 15701

State University of R.716

Jowa Libraries

Serials Acquisition

Iowa City, Iowa 52240

James E. Irby R-657

Assoc. Prof. of Span. \& Port.

Princeton Univ.

Princeton, N.J. 08540

Didier $T$. Jaen R-553

Spanish Department

University of California

Davis, California 95616

Mr. Orlandn Jardini R-605

77 Owendale Ave.

Pittsburgh, Pa. 15274

Tosé Olivio Jiménez R.554

392 Central Park West (20-T)

New oYtk, N.Y. 10025

*Ernest A. Johnson R-602

195 West St.

Amherst, Mass.

W. Thomas Judd R-893

4690 Haverhill Rd.

Detroit, Michigan 48224

Sonja Karsen R-555

Dept. of Modern Languages

Skidmore College

Saratoga Springs, N.Y. 12866
Robert B. Knox R-556

Dept. of Foreign Languages

Washington State University

Pullman, Wash. 99163

Phillip Koldewyn R-223

Asst. Prof. of Spanish

Claremont Men's College

Claremont, California 91717

Prof. Anthony Lamb. R-558

Western Illinois Univ.

C-10 507 W. Adams St.

Macomb, Illinois 61455

Gloria Aledort Levine R-028

Associate Professor,

The Dept. of Modern Langs.

Oregon State University

Corvallis, Oregon 97331

Myron E. Lichblau R-559

Hall of Languages

Syracuse University

Syracuse. N.Y.

Carlos Lozano R-560

Dept. of Romance Langs.

Saint Mary's College

Saint Mary's College, Calif,

Vera Maslow R-562

315 W. 57th Street

The Towers

New York, 19, N.Y.

Malcolm D. McLean R-565

2555 Cockrell

Fort Worth, Texas 76109

*Prof. Robert G. Mead, Jr. Dept. of Romance Languages University of Conneticut

Storrs, Conn. 06268

*W. L. Meinhardt R-599

Dept. of Span., Ital,, Port.

University of Ill.

Utbana, Ill. 61803

George Melnvkovish

Dept. of Hisp. Langs.

University of Pittsburgh

Pittsburgh, Pa. 15213

Serge Mitrofanoff R-609

715 So. Negley Ave.

Pittsburgh, Pa. 15232

Luis Monguio R-603

Dept. of Spanish \& Port.

University of California

Berkeley, California 94720 
Prof. Casper J. Moresello R-563

Dept. of Romance Languages

Wesleyan University

Middletown, Conn. 06457

*Robert J. Morris R-781

Dept. of Span. \& Italian

Univ. of Kentucky-Bradley Hall

Lexington, Kentucky 40503

Spanish Department R-520

c/o Miss Leonor Andrade

Mount Mary College

Milwaukee. Wisconsin 53222

Edwin C. Munro R-667

State University of N.Y.

1223 Western Ave.

Albany, New York

*Prof. Frederic W. MurrayR-931

Dept. of Foreing Langs \& Clas.

Clarence Cook Little Hall

University of Maine

Orono, Maine 04473

Marshall R. Nason R-566

Director, Lang. \& Area

Center

University of New Mexico

Albuquerque, New Mexico

Mrs. Zoila E. Nelken R-282

37 Sixth Avenue

San Francisco, California

Graciela P. Nemes R-567

6926 Pineway

Hyattsville, Maryland 20782

CPO3478

Nevada Southern

University Libraty

Periodicals

Las Vegas, Nevada 89109

Prof. Enrique Noble R-600

Dept. of Modern langs.

University of Mo.-St. Lauis

8001 Natural Bridge Rd.

St. Louis, Mo. 63121

Otto Olivera R-569

Dept. of Spanish Langs.

Tulane University

New Orleans, La.
Dr. Betty Tyree Osiek R-758

Foreign Languages \&

Literatures

Southern Illinois University

Edwardsville, Illinois 62025

Marta L. Pérez R-571

6655 Marblow Dr.

Jacksonville, Fla. 22211

Prof. Andrea I. Pineda de Camps

Dept. of Romance \& Ger. Langs.

Wayne State University

Detroit, Michigan

Helena Ponseti R-598

315 Ellis Ave.

Iowa City, Iowa

Library R-638

Princeton University

Princeton, N.J.

Prof. Manuel D. Ramirez R- 573

Dept. of Modern Foreign Langs.

University of Georgia

Athens. Ga.

Susana Redonde de Feldman R-541

445 Riverside Drive

New York, N.Y. 10027

Richard M. Reeve R-643

Dept. of Spanish \& Port.

Univ. of Calif, L.A.

Los Angeles, Calif. 90025

Carlos Ripoll R-572

Dept. of Spanish

Oueens College

Flushing 67, New York

Prof. Tames Willis Robb. R-575

George Washington Univ.

Washington, 6, D.C.

Renato Rosaldo R-576

Debt. of Romance Langs.

University of Arizona

Tucson, Arizona

Dr. Ivan A. Schulman R-579

Washington University

Department of Spanish

St. Louis, Missouri 63130
Mrs. Lee R. Shaw R-88.2

940 Talley Rodd

Chattanooga, Tenn. 37411

John D. Smith R-368

Spanish Department

San Diego State College

San Diego, California 92115

Mario T. Soria R-782

4238 Colony Road

Cleveland, Ohio 44121

Raymond D. Souza R-578

1409 W. 22nd St.

Lawrence, Kans. 66044

Martin C. Taylor R.580

Department of Spanish

University of California

Berkeley, California 94720

Edward D. Terry R-581

P.O. Box 1911

University of Alabama

University, Alabama 35486

Library R-583

Trinity College

Hartford, Conn. 06106

*E. Daymond Turner R-632

Dept. of Foreign Langs.

P.O. Box 12665

Charlotte, North Carolina 28205

A. Valbuena (Briones)

203 Nottingham Road

Neward, Delaware 19711

Luis Valdespino R.405

Co-ordinator of the Span. Prog.

Central Washington State

Col.

Ellensburg, Washington

Charles M. Vance R-406

997 Caldwell Lane

Nashville, Tennessee 37204

* Ray Verzascani R-408

Dept. of Modem Languages

Oregon State University

Corvallis, Oregon 97331

*Dr. Grace E. Weeks R.588

Samford University

Birmingham, Ala. 35209 
Library R-589

Wellesley College

Wellesley, Mass.

Library R-590

Williams College

Williamstown, Mass.

*Margarita Winikoff R-613

709 Washington Drive

Pittsburgh, Ph. 15229

Miss Ruth Wold R-435

Dept. of Foreign Languages

San Fernando Valley State

Col.

North Ridge, California

Dordld A. Yates R-441

537 Wells Hall

Michigan State University

E. Lansing, Michigan

Juan C. Zamora R-591

State University of N.Y.

Dept. of Modern Languages

Buffalo 14. N.Y.

Dr. F. J. Zilli, Chairman R-822

Foreign Language Department

Southern Cannecticut State

Col.

501 Crescent Street

New Haven, Conn. 06515
CANADA

EL SALVADOR

"Keith Ellis R-887

Dept. of Ital. \& Hispanis Studies

University of Toronto

Toronto, Canadá

Prof. Richard L. Jackson R-854

Dept. of Spanish

Carleton University

Ottawa 1, Canadá

* Prof. Robert J. Glickman R-625

Dept. of Ital. \& Hisp. Studies

University of Toronto

Toronto 5, Canada

Prof. Ross F. Larson R-451

Dept. of Spanish

Carleton University

Ottawa 1, Ontario Canadá

Fernando de Toro Garland R-544

Dept. of Modern Languages

Carleton University

Ottawa, Ontario Canadá

DOMINICAN REPUBLIC

Carlos Fernández-Rocha

Bibliotecario Antillense

Apartado 701

Santiago de los Caballeros

República Dominicana
Hum. \& Soc. Sci. R-711

Biblioteca del Centro

Udn de Occidente

San Salvador

El Salvador, Cen. América

Hum. \& Soc. Sci. R-710

Biblioteca del Centro

Un de Oriente

San Salvador

El Salvador. Cen. América

\section{PUERTO RICO}

Antillian College, Bib. R-5i2

Colegio Adventista

Puertorriqueño

P.O. Box 118

Mayagüiez, Puerto Rico

Edna Coll R-499

Departamento de Español

Recinto Univ. de Mayagüez

5053 College Station

Mayagïez, Puerto Rico

Angel Luis Morales R-501

Universidad de Puerto Rico

Dept. of Estudios Hispánicos

Rio Piedras, Puerto Rico

Elia SuIsona R-686

Oficial de Relaciones Públicas

de la Saciedad de Autores

Apartado 21324

Universidad de Puerto Rico

Río Piedras, Puerto Rico 00911

\section{VENEZUELA}

Instituto Pedagógico R-811

Experimental

Barquisimeto

Estado Lara-Venezuela 
